Article

\title{
Solving Multi-Point Boundary Value Problems Using Sinc-Derivative Interpolation
}

\author{
Kenzu Abdella ${ }^{1, *,+}$ (D) and Jeet Trivedi ${ }^{2,+}$ \\ 1 Department of Mathematics, Statistics and Physics, Qatar University, Doha 2713, Qatar \\ 2 Department of Mathematics, Western University, London, ON N6A 5B7, Canada; jtrived4@uwo.ca \\ * Correspondence: kabdella@qu.edu.qa \\ + These authors contributed equally to this work.
}

Received: 21 May 2020; Accepted: 8 June 2020; Published: 25 November 2020

\begin{abstract}
In this paper, the Sinc-derivative collocation method is used to solve linear and nonlinear multi-point boundary value problems. This is done by interpolating the first derivative of the unknown variable via Sinc numerical methods and obtaining the desired solution through numerical integration of the interpolation and all higher order derivatives through successive differentiation of the interpolation. Non-homogeneous boundary conditions are reduced to homogeneous using suitable transformations. The efficiency and the accuracy of the method are tested using illustrative examples previously considered by other researchers who used different approaches. The results show the excellent performance of the Sinc-derivative collocation method.
\end{abstract}

Keywords: multi-point boundary value problems; sinc-collocation; sinc-derivative; numerical methods; differential equations

\section{Introduction}

Due to their numerous applications in science and engineering, multi-point boundary value problems (MPBVPs) continue to attract considerable research interests [1-4]. MPBVPs constitute boundary value problems consisting of differential equations which are subjected to boundary conditions involving the boundary data of the subdomains of the overall domain under consideration. In terms of their applications, MPBVPs have been used to model a wide range of real world problems including, the optimal design of large size bridges which are contrived with multi-point supports [5], the vibration of a guy wire of uniform cross-section with multiple parts of varying densities [6], the flow of fluids through ground layers in which each layer can be considered a subdomain [7], the deformation of beams and plate deflection theory $[5,7,8]$ and many problems involving the theory of elastic stability [9].

The existence of solutions and other theoretical aspects of MPBVPs have been studied by many researchers [10-15]. Since there are no analytic solutions of most MPBVPs, numerical methods are used to approximate the exact solutions. These include the adaptive finite difference method [16], the shooting method [17], the domain decomposition method [18], the Hes variational iteration method [5], the Chebyshev series [19], semi-analytical methods [20], the Homotopy method [21], the reproducing Hilbert kernel space method [22-26] and the Sinc-collocation method [8].

In this paper we use the Snc-derivative collocation numerical method to solve the multi-point boundary value problem of the form

$$
y^{(n)}=g\left(x, y, y^{\prime}, \cdots, y^{(n-1)}\right), \quad x \in[a, b]
$$


for which the boundary conditions are given as the value of the function $y$ or its derivatives or combination of them at $m$ boundary points of the subdomains within the domain $[a, b]$. Therefore, the boundary conditions are of the form:

$$
\sum_{j=1}^{m} \sum_{i=0}^{n-1} a_{i, j}^{k} y^{(i)}\left(\xi_{j}\right)=r_{k}, \quad k=1, \cdots, n,
$$

where $a \leq \xi_{1}<\xi_{2}<\cdots<\xi_{m} \leq b, a_{i, j}$ and $r_{k}$ are real constants. It is assumed that the function $g$ has the properties which guarantee the existence and uniqueness of the solution of the problem.

Sinc numerical methods have been used to solve a wide range of applications involving boundary value problems [27-34]. In addition to their high efficiency in handling boundary value problems involving singularities, Sinc numerical methods are highly accurate since approximate solutions based on Sinc bases are characterized by exponentially decaying errors [35-38]. Recently, Saadatmandi and Dehghan showed that the Sinc-collocation technique can be very effective in solving multi-point boundary value problems numerically [8]. By converting the MPBVPs into discrete system of equations via Sinc interpolation, they showed that exponentially converging solutions can be obtained. While the conventional strategy in implmenting Sinc method to solve boundary value problems is to interpolate the unknown variable and to use successive differentiation to obtain higher derivatives, the Sinc-derivative collocation approach is to interpolate the derivative of the unknown function and to obtain the unknown function via numerical integration [39]. The Sinc-derivative collocation approach has the advantage of averaging and damping out the numerical errors inherently present in the computation of the derivatives [39,40]. By enhancing the accuracy of the approximation of the derivative of the unkown function the Sinc-derivative collocation approach has been shown to result in more accurate solutions than those obtained using the conventional approach [41,42].

In this paper, we use the Sinc-derivative collocation method to obtain numerical solution of second order MPBVPs in which nonhomogeneous boundary conditions are treated via appropriate transformations which reduces them to homogeneous ones. The proposed approach is tested using several illustrative examples that have been considered in earlier literatures that use other numerical methods. The results show that the proposed method is very efficient and highly accurate.

Following a brief description of the Sinc-collocation method in Section 2, the Sinc-derivative collocation approach for MPBVPs is presented in Section 3. Finally, illustrative examples that demonstrate the accuracy of the proposed method and brief concluding remarks are presented in Section 4 and Section 5 respectively.

\section{Sinc Preliminaries}

The sinc function is defined by

$$
\operatorname{Sinc}(x)= \begin{cases}\frac{\sin (\pi x)}{\pi x} & \text { if } x \neq 0 \\ 1 & x=0\end{cases}
$$

For a function $f: \mathbb{R} \rightarrow \mathbb{R}$, for a step size $h>0$, the series

$$
C(f, h)(x)=\sum_{k=-\infty}^{\infty} f(k h) S(k, h)(x)
$$

where $S(k, h)(x)$ is the translated $k^{\text {th }}$ Sinc function given by

$$
S(k, h)(x)=\operatorname{Sinc}\left(\frac{x-k h}{h}\right)
$$


is called the Whittaker Cardinal expansion of $f$ when the series converges [36]. For computation purposes, the Sinc approximation is obtained by truncating the infinite series using $2 N+1$ collocation points as

$$
C_{N}(f, h)(x) \equiv \sum_{k=-N}^{N} f(k h) S(k, h)(x)
$$

for a given positive integer $N$. For a class of functions with specific growth restrictions, the Sinc approximations exhibit exponentially decaying absolute errors as established by the theorems subsequent to the following definition.

Definition 1. Let $D_{d}$ denote the infinite strip of width $2 d(d>0)$ in the complex plane:

$$
D_{d}=\left\{z=x+i y|| y \mid<d<\frac{\pi}{2}\right\} .
$$

Then $B\left(D_{d}\right)$ is defined as a the class of functions $f$ that are analytic in $D_{d}$ such that

$$
N\left(f, D_{d}\right) \equiv \lim _{\epsilon \rightarrow 0} \int_{\partial D_{d}(\epsilon)}|f(z)||d z|<\infty
$$

where for $0<\epsilon<1$,

$$
D_{d}(\epsilon)=\left\{z=x+i y|| x\left|<\frac{1}{\epsilon},\right| y \mid<d(1-\epsilon)\right\} .
$$

and $\partial D_{d}$ represents the boundary of $\partial D_{d}$.

For functions that decay single exponentially, we have the following theorem, due to Stenger [35].

Theorem 1 (Stenger [35]). If $f(x) \in B\left(D_{d}\right)$ and decays single exponentially for $x \in \mathbb{R}$, that is

$$
|f(x)| \leq \beta \exp (-\alpha|x|) \text { for all } x \in \mathbb{R}
$$

where $\beta$ and $\alpha$ are positive constants, then the error of the Sinc approximation is bounded by:

$$
\sup _{-\infty \leq x \leq \infty}\left|f(x)-\sum_{k=-N}^{N} S(k, h)(x) f(k h)\right| \leq C \sqrt{N} \exp (-\sqrt{\pi d \alpha N})
$$

for some positive constant $C$ and where the mesh size $h$ for the Sinc-collocation is taken as:

$$
h=\sqrt{\frac{\pi d}{\alpha N}} .
$$

While for double exponentially decaying functions, we have the following theorem due to Sugihara [43].

Theorem 2 (Sugihara [43]). If $f(x) \in B\left(D_{d}\right)$ and decays double exponentially for $x \in \mathbb{R}$, that is,

$$
|f(x)| \leq \beta \exp (-\alpha \exp (\gamma|x|)) \text { for all } x \in \mathbb{R}
$$


where $\alpha, \beta$ and $\gamma$ are positive constants, then the error of the Sinc approximation is bounded by:

$$
\sup _{-\infty \leq x \leq \infty}\left|f(x)-\sum_{k=-N}^{N} S(k, h)(x) f(k h)\right| \leq C \exp \left(\frac{-\pi d \gamma N}{\log (\pi d \gamma N / \alpha)}\right)
$$

for some positive constant $C$ and where the mesh size $h$ is taken as:

$$
h=\frac{\log (\pi d \gamma N / \alpha)}{\gamma N} .
$$

In order to use these interpolations on a finite interval $[a, b]$, we use a variable transform $\phi:[a, b] \rightarrow \mathbb{R}$ with an associated inverse transform $\psi: \mathbb{R} \rightarrow[a, b]$. Combined with the variable transform, the truncated cardinal expansion of a function over an interval $[a, b]$ can be written as

$$
C(f, h)_{N}(x)=\sum_{k=-N}^{N} f(\psi(k h))(S(k, h) \circ \phi)(x)
$$

The theorems below which follow directly from Theorems 1 and 2 above quantify the error bound associated with this interpolation $[35,43]$.

Theorem 3. If $x=\psi(\xi)$ and $f(\psi(\xi)) \in B\left(D_{d}\right)$ and decays single exponentially for $\xi \in \mathbb{R}$, that is

$$
\left.\left|f(\psi(\xi)) \psi^{\prime}(\xi)\right| \leq \beta \exp (-\alpha|\xi|)\right) \quad \forall \xi \in \mathbb{R}
$$

where $\beta$ and $\alpha$ are positive constants, then the error of the Sinc approximation is bounded by:

$$
\sup _{-\infty \leq x \leq \infty}\left|f(x)-\sum_{k=-N}^{N} f(\psi(k h))(S(k, h) \circ \phi)(x)\right| \leq C \sqrt{N} \exp (-\sqrt{\pi d \alpha N})
$$

for some positive constant $C$ and where the mesh size $h$ for the Sinc-collocation is taken as:

$$
h=\sqrt{\frac{\pi d}{\alpha N}} .
$$

Theorem 4. If $x=\psi(\xi)$ and $f(\psi(\xi)) \in B\left(D_{d}\right)$ and decays double exponentially for $\xi \in \mathbb{R}$, that is,

$$
\left|f(\psi(\xi)) \psi^{\prime}(\xi)\right| \leq \beta \exp (-\alpha \exp (\gamma|\xi|)) \quad \forall \xi \in \mathbb{R}
$$

where $\alpha, \beta$ and $\gamma$ are positive constants, then the error of the Sinc approximation is bounded by:

$$
\sup _{-\infty \leq x \leq \infty}\left|f(x)-\sum_{k=-N}^{N} f(\psi(k h))(S(k, h) \circ \phi)(x)\right| \leq \operatorname{Cexp}\left(\frac{-\pi d \gamma N}{\log (\pi d \gamma N / \alpha)}\right)
$$

for some positive constant $C$ and where the mesh size $h$ is taken as:

$$
h=\frac{\log (\pi d \gamma N / \alpha)}{\gamma N} .
$$


The frequently used single exponential transformations $\phi_{S}(x)$ is given by [35]

$$
\xi=\phi_{S}(x)=\log \left(\frac{x-a}{b-x}\right)
$$

with the corresponding inverse:

$$
x=\psi_{S}(\xi)=\frac{b+a}{2}+\frac{b-a}{2} \tanh (\xi) .
$$

Similarly the double exponetial transformation $\phi_{D}(x)$ is given by [37]

$$
\xi=\phi_{D}(x)=\log \left(G+\sqrt{G^{2}+1}\right), G=\frac{1}{\pi} \phi_{S}(x)
$$

with the corresponding inverse:

$$
x=\psi_{D}(\xi)=\frac{b+a}{2}+\frac{b-a}{2} \tanh \left(\frac{\pi}{2} \sinh (\xi)\right) .
$$

\section{The Sinc-Derivative Method for MPBVPs}

Consider the problem stated in Equation (1) along with its boundary conditions for $n=2$. Higher order cases can be treated similarly. For $n=2$, the boundary conditions involve first order derivative of the unknown function.

$$
\begin{gathered}
y^{\prime \prime}=g\left(x, y, y^{\prime}\right), \quad x \in[a, b] \\
\sum_{j=1}^{m}\left(a_{0, j}^{1} y\left(\xi_{j}\right)+a_{1, j}^{1} y^{\prime}\left(\xi_{j}\right)\right)=r_{1}, \\
\sum_{j=1}^{m}\left(a_{0, j}^{2} y\left(\xi_{j}\right)+a_{1, j}^{2} y^{\prime}\left(\xi_{j}\right)\right)=r_{2},
\end{gathered}
$$

Following the Sinc-derivative interpolation approach, we approximate the derivative of the solution as

$$
y_{N}^{\prime}(x)=u_{N}^{\prime}(x)+p^{\prime}(x)
$$

where $u_{N}^{\prime}(x)$ is the collocation part of the solution's derivative which is given by:

$$
u_{N}^{\prime}(x)=\sum_{k=-N}^{N} c_{k}(S(k, h) \circ \phi)(x)
$$

where the $c_{k} \mathrm{~s}$ are $2 N+1$ unknown Sinc coefficients that need to be determined and $p(x)$ is a quadratic polynomial whose derivative is defined as:

$$
p^{\prime}(x)=c_{N+1}\left(\frac{b-x}{b-a}\right)+c_{N+2}\left(\frac{x-a}{b-a}\right)
$$

which after integration yields:

$$
p(x)=c_{N+1}\left(\frac{(b-a)^{2}-(b-x)^{2}}{2(b-a)}\right)+c_{N+2}\left(\frac{(x-a)^{2}}{2(b-a)}\right)+c_{N+3}
$$


where we define

$$
c_{N+1}=y_{N}^{\prime}(a), \quad c_{N+2}=y_{N}^{\prime}(b), \quad \text { and } \quad c_{N+3}=y_{N}(a) .
$$

Note that Equations (17) and (18) imply that:

$$
p^{\prime}(a)=c_{N+1}=y_{N}^{\prime}(a), \quad p^{\prime}(b)=c_{N+2}=y_{N}^{\prime}(b), \quad \text { and } \quad p(a)=c_{N+3}=y_{N}(a) .
$$

Hence, by Equation (15), this implies that the Sinc collocation part, $u_{N}^{\prime}(x)$ satisfies the homogenous boundary conditions at the boundary end points:

$$
u_{N}^{\prime}(a)=u_{N}^{\prime}(b)=0 .
$$

Hence when the the boundary conditions of the MPBVP are evaluated, these homogeneous conditions on $u_{N}^{\prime}$ will be imposed. This particular approach of transforming the MPBVP into a homogeneous boundary conditions has been used by other researchers [44,45].

Evaluating the expression in (16) at the collocation points given by

$$
x_{l}=\psi(l h) \quad l=-N, \ldots, N
$$

gives

$$
u_{N}^{\prime}\left(x_{l}\right)=\sum_{k=-N}^{N} c_{k} \delta_{l, k}^{(0)}
$$

where

$$
\delta_{k, l}^{(0)}=\left\{\begin{array}{lll}
1 & \text { if } & k \neq l \\
0 & \text { if } & k=l
\end{array}\right.
$$

which yields

$$
u_{N}^{\prime}\left(x_{l}\right)=c_{l}
$$

From here, we can approximate all the higher derivatives by differentiating Equation (15). For example the second derivative, $y_{N}^{\prime \prime}(x)$ is calculated as follows:

$$
\begin{aligned}
y_{N}^{\prime \prime}(x) & =\sum_{k=-N}^{N} c_{k} \frac{d}{d x}((S(k, h) \circ \phi)(x))+p^{\prime \prime}(x) \\
& =\sum_{k=-N}^{N} c_{k} \frac{d}{d \phi}((S(k, h) \circ \phi)(x)) \phi^{\prime}(x)+p^{\prime \prime}(x) .
\end{aligned}
$$

Hence at the Sinc nodes $x_{l}$

$$
y_{N}^{\prime \prime}\left(x_{l}\right)=\sum_{k=-N}^{N} c_{k} \delta_{k, l}^{(1)} \phi^{\prime}\left(x_{l}\right)+p^{\prime \prime}\left(x_{l}\right)
$$

where

$$
\frac{d}{d \phi}\left(\left.(S(k, h) \circ \phi)(x)\right|_{x=x_{l}}=\delta_{k, l}^{(1)}=\frac{1}{h} \begin{cases}0 & k=l \\ \frac{(-1)^{l-k}}{(l-k)} & k \neq l .\end{cases}\right.
$$

Note that higher order equations are treated similarly where we can use the general expressions as follows [27]: 


$$
\begin{array}{r}
\frac{d^{2 r}}{d \phi^{2 r}}\left(\left.(S(k, h) \circ \phi)(x)\right|_{x=x_{l}}=\delta_{k, l}^{(2 r)}=\frac{1}{h^{2 r}} \begin{cases}\frac{(-1)^{r} \pi^{2 r}}{2 r+1} & k=l \\
\frac{(-1)^{l-k}}{(l-k)^{2 r}} \sum_{s=0}^{r-1} \frac{(-1)^{(s+1)}(2 r) !}{(2 s+1) !} \pi^{2 s}(l-k)^{2 s} & k \neq l\end{cases} \right. \\
\frac{d^{2 r+1}}{d \phi^{2 r+1}}\left(\left.(S(k, h) \circ \phi)(x)\right|_{x=x_{l}}=\delta_{k, l}^{(2 r+1)}=\frac{1}{h^{2 r+1}} \begin{cases}0 & k=l \\
\frac{(-1)^{l-k}}{(l-k)^{2 r}} \sum_{s=0}^{r} \frac{(-1)^{(s)}(2 r+1) !}{(2 s+1) !} \pi^{2 s}(l-k)^{2 s} & k \neq l\end{cases} \right.
\end{array}
$$

with $r=1,2,3, \ldots$

The expression for the solution $y_{N}(x)$ is obtained by integrating Equation (15) [39]:

$$
y_{N}(x)=\int_{a}^{x} u_{N}^{\prime}(s) d s+p(x) .
$$

Hence

$$
\begin{aligned}
y_{N}\left(x_{l}\right) & =\int_{a}^{x_{l}} u_{N}^{\prime}(s) d s+p\left(x_{l}\right) \\
& =\int_{a}^{x_{l}} \sum_{k=-N}^{N}\left(c_{k}(S(k, h) \circ \phi)(s)\right) d s+p\left(x_{l}\right) \\
& =\sum_{k=-N}^{N} c_{k} \int_{a}^{x_{l}}((S(k, h) \circ \phi)(s)) d s+p\left(x_{l}\right) \\
& =\sum_{k=-N}^{N}\left(\frac{c_{k} \delta_{l, k}^{(-1)}}{\phi^{\prime}\left(x_{k}\right)}\right)+p\left(x_{l}\right)
\end{aligned}
$$

where

$$
\delta_{l, k}^{(-1)}=h \begin{cases}\frac{1}{2}+\frac{\operatorname{Si}(\pi(l-k))}{\pi} & \text { if } k \neq l \\ \frac{1}{2} & \text { if } k=l\end{cases}
$$

and $\operatorname{Si}(z)$ is the Sine Integral given by

$$
\operatorname{Si}(z)=\int_{0}^{z} \frac{\sin (t)}{t} d t
$$

Therefore, the unknowns that must be determined include the $2 N+4, c_{k}$ s for $k=-N \ldots N+3$. Applying the discretization in Equation (1) at the $2 N+1$ Sinc nodes and at the two boundary equations given by Equations (13) and (14), we obtain a total of $2 N+3$ equations. Therefore, we need an additional equation in order to solve for the $2 N+4$ unknowns. This is done by applying a discretization at an additional Sinc point, $x_{-N-1}$. While applying the boundary conditions of Equations (13) and (14), we note that:

$$
\begin{aligned}
y_{N}(a) & =c_{N+3} \\
y_{N}(b)=\int_{a}^{b} u_{N}^{\prime}(s) d s+p(b) & =\sum_{k=-N}^{N} h\left(\frac{c_{k}}{\phi^{\prime}\left(x_{k}\right)}\right)+p(b) .
\end{aligned}
$$


and $y_{N}^{\prime}\left(\xi_{j}\right)$ and $y_{N}\left(\xi_{j}\right)$ are evaluated using Equations (15) and (30) respectively as follows:

$$
\begin{gathered}
y_{N}\left(\xi_{j}\right)=\int_{a}^{\xi_{j}} u_{N}^{\prime}(s) d s+p\left(\xi_{j}\right)=\sum_{k=-N}^{N} h\left(\frac{c_{k} \delta_{k}^{(-j)}}{\phi^{\prime}\left(x_{k}\right)}\right)+p\left(\xi_{j}\right) . \\
y_{N}^{\prime}\left(\xi_{j}\right)=u_{N}^{\prime}\left(\xi_{j}\right)+p^{\prime}\left(\xi_{j}\right)
\end{gathered}
$$

where

$$
\delta_{k}^{(-j)}= \begin{cases}\frac{1}{2}+\frac{\operatorname{Si}\left(\frac{\pi}{h}\left(\phi\left(\xi_{j}\right)-k h\right)\right)}{\pi} & \text { if } k \neq j \\ \frac{1}{2} & \text { if } k=j\end{cases}
$$

\section{Numerical Illustrations}

In order to demonstrate the enhanced rate of convergence and accuracy of the Sinc-derivative collocation approach with respect to other approaches, we solve the following problems considered in the recent literature. All the computations of the Sinc-derivative results are performed using Mathematica 11.3.

Example 1. Consider the following nonlinear MPBVP solved in [8] using regular Sinc-collocation approach.

$$
y^{\prime \prime}(x)+\left(x^{3}+x+1\right) y^{2}(x)=f(x) \quad x \in[0,1]
$$

with boundary conditions,

$$
\begin{aligned}
& y(0)=\frac{1}{6} y\left(\frac{2}{9}\right)+\frac{1}{3} y\left(\frac{7}{9}\right)-0.0286634 \\
& y(1)=\frac{1}{5} y\left(\frac{2}{9}\right)+\frac{1}{2} y\left(\frac{7}{9}\right)-0.0401287
\end{aligned}
$$

where

$$
f(x)=\frac{1}{9}\left[-6 \cos \left(x-x^{2}\right)+\sin \left(x-x^{2}\right)\left(-3\left(1-2 x^{2}\right)+\left(1+x+x^{3}\right) \sin \left(x-x^{2}\right)\right)\right]
$$

The exact solution to this MPBVP is given by $y(x)=\frac{1}{3} \sin \left(x-x^{2}\right)$.

The plot of the absolute errors of the Sinc-derivative solution described in Section 3 is depicted in Figure 1. for $N=20$ and $N=40$. The result is highly accurate with a maximum absolute errors of $2.6 \times 10^{-14}$ for $N=20$ and $6.7 \times 10^{-22}$ for $N=40$. For $N=20$ the result reported in [8] which uses the regular Sinc-collocation method has a maximum absolute error of $2 \times 10^{-7}$. The best results reported for this example in $[5,26]$ have maximum absolute errors of $7.6 \times 10^{-10}$ and $3.5 \times 10^{-12}$ respectively. 


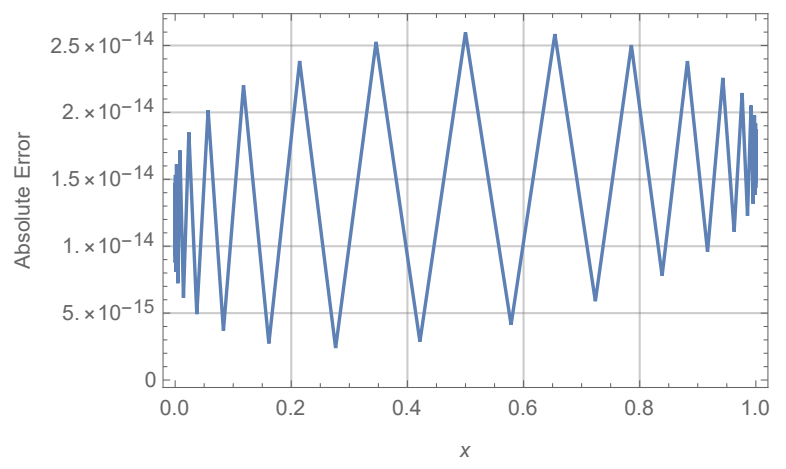

(a) $N=20$

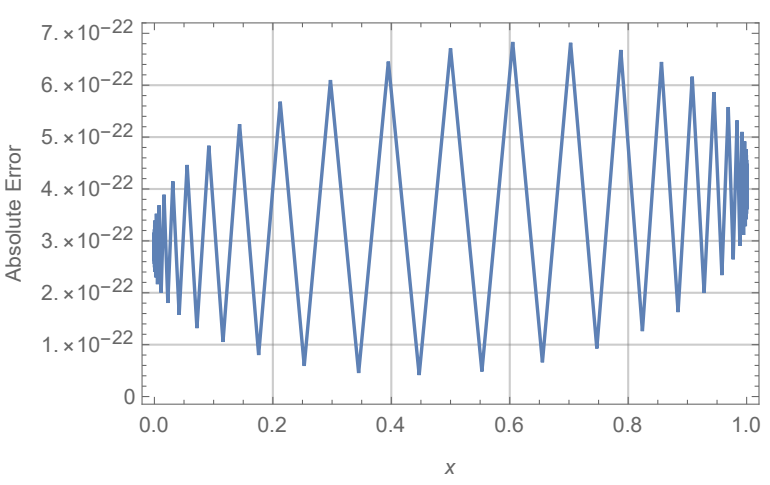

(b) $N=40$

Figure 1. Plots of the absolute error, for example, 1, using the Sinc-derivative interpolation for (a) $N=20$ and (b) $N=40$.

The exponential convergence of the method described in this paper is illustrated in Figure 2. where the logarithmic plot of the maximum error as a function of the number of nodes $N$ shown. Both the regular Sinc-collocation method reported in [8] and the current method are displayed. For the Sinc-collocation method, only the portion of the graph that was reported in [8] is displayed. The plot confirms that the Sinc-derivative approach is a highly accurate method for solving MPBVPs.

Example 2. Consider the following nonlinear MPBVP solved in $[46,47]$ using techniques that are based on the reproducing kernel method.

$$
x(x-1) y^{\prime \prime}(x)+6 y^{\prime}(x)+2 y(x)+y^{2}(x)=f(x) \quad x \in[0,1]
$$

with boundary conditions,

$$
\begin{aligned}
& y(0)+y\left(\frac{2}{3}\right)=\sinh \left(\frac{2}{3}\right) \\
& y(1)+y\left(\frac{4}{5}\right)=\frac{1}{2} \sinh \left(\frac{4}{5}\right)+\sinh (1)
\end{aligned}
$$

where

$$
f(x)=6 \cosh (x)+\sinh (x)\left(2+x-x^{2}+\sinh (x)\right) .
$$

The exact solution to this MPBVP is given by $y(x)=\sinh (x)$. 


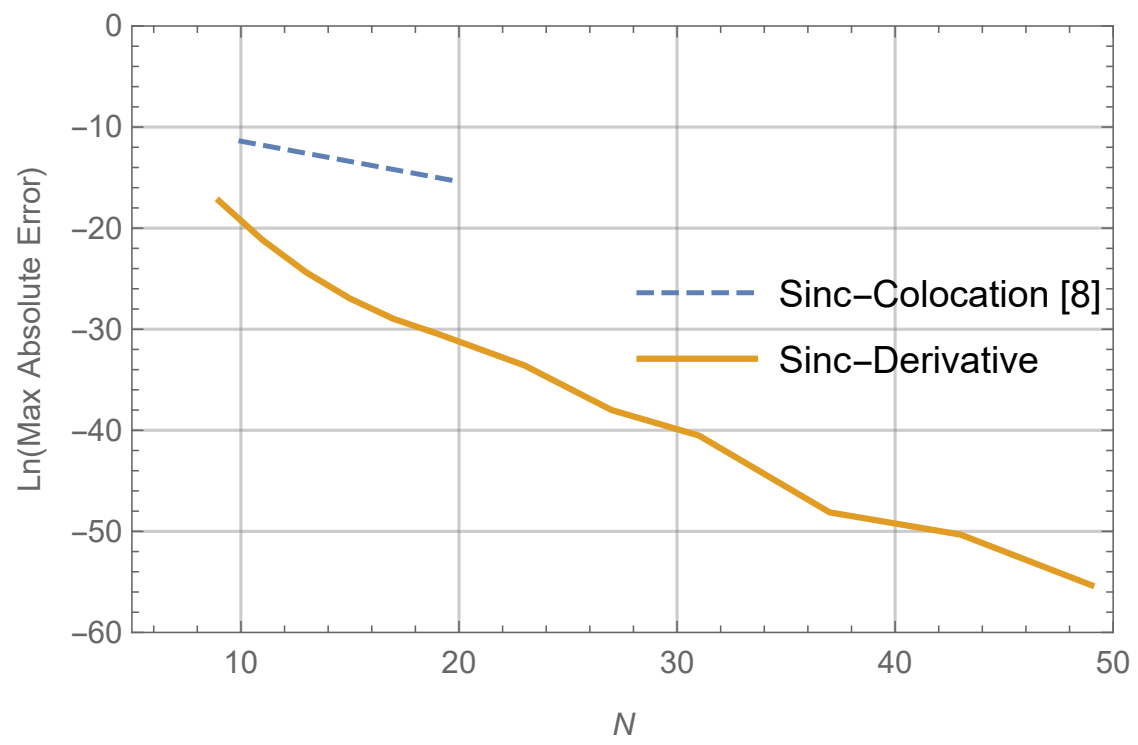

Figure 2. Log of the max absolute errors for the method used in [8] and the current Sinc-derivative method as a function of the number of nodes $(N)$, for example, 1 .

The plot of the absolute errors of the Sinc-derivative solution described in Section 3 is depicted in Figure 3. for $N=20$ and $N=40$. The maximum absolute errors are $1.6 \times 10^{-12}$ for $N=20$ and $8.1 \times 10^{-20}$ for $N=40$. The results reported for this example in [46] and [47] have maximum absolute errors of $3.1 \times 10^{-6}$ and $2.5 \times 10^{-5}$ respectively. The present method clearly performs well.

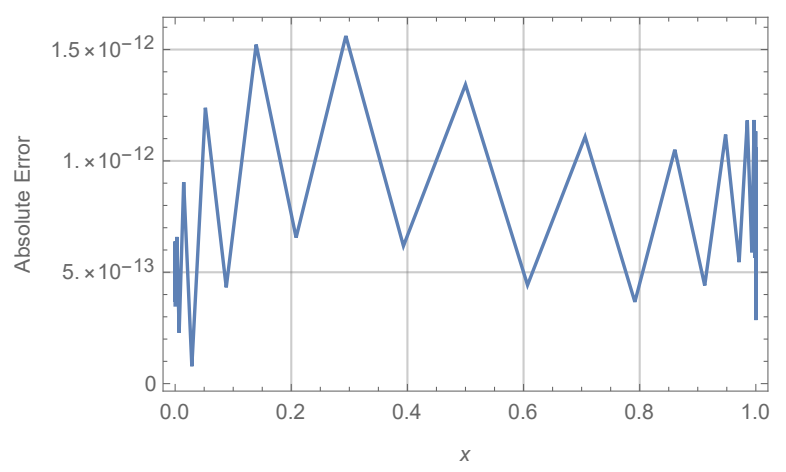

(a) $N=20$

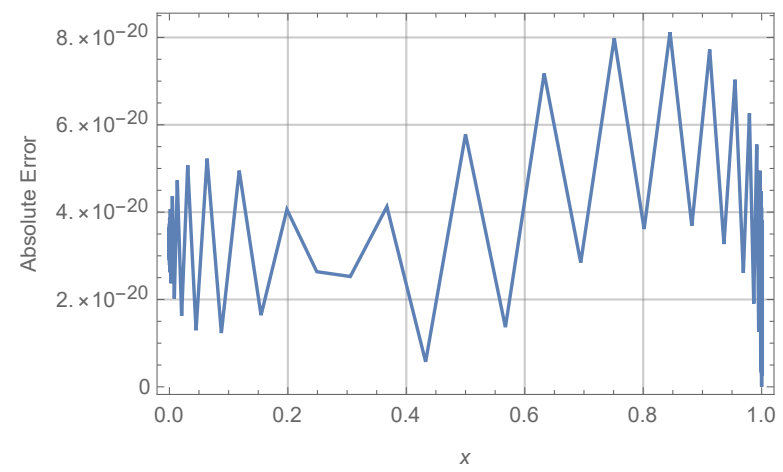

(b) $N=40$

Figure 3. Plots of the absolute error, for example, 2, using the Sinc-derivative interpolation for (a) $N=20$ and (b) $N=40$.

Example 3. Consider the following linear third order MPBVP solved in [48] using techniques that are mainly based on the the least square approximation method, the Lagrange-multiplier method and the residual error function method. It is also solved in [8] using regular Sinc-collocation method. Other Numerical solutions of this example are reported in [21,49-51].

$$
y^{(3)}(x)-k^{2} y^{\prime}(x)+a=0,
$$

with the following three-point boundary conditions,

$$
y^{\prime}(0)=y^{\prime}(1)=0, y\left(\frac{1}{2}\right)=0 .
$$


This MPBVP was obtained by Krajcinvic [52] in the analysis of sandwich beams. When subjected to a uniformly distributed load along the entire length, the distribution of shear deformation is given by Equation (47) where $k$ and $a$ represent physical constants that depend on the elastic properties of the lamina. The first two boundary conditions in Equation (48) represent a zero-shear bi-moment conditions at each ends. The third condition is obtained from symmetry consideration.

The exact solution to this MPBVP is given by:

$$
y(x)=\frac{a}{k^{3}}\left(\sinh \left(\frac{k}{2}\right)-\sinh (k x)\right)+\frac{a}{k^{2}}\left(x-\frac{1}{2}\right)+\frac{a}{k^{3}}\left(\cosh (k x)-\cosh \left(\frac{k}{2}\right)\right) \tanh \left(\frac{k}{2}\right) .
$$

We solve this using Sinc-derivative interpolation for the case $k=5$ and $a=1$. Since this is a third order MPBVP we have a third boundary condition which gives us the $2 N+4$ th equation. Hence unlike the second order case there will be no need to discretize the differential equation at the extra $-N-1$ nodal point.

The plot of the absolute errors of the Sinc-derivative solution for Example 3. is depicted in Figure 4. for $N=5, N=10, N=20$ and $N=25$.

The maximum absolute errors are $1.6 \times 10^{-11}$ for $N=20$ and $4.8 \times 10^{-13}$ for $N=25$. The results reported for this example in [8] have maximum absolute errors of $5.0 \times 10^{-7}$ and $6.8 \times 10^{-8}$ for $N=20$ and $N=25$ respectively. The best result presented in [52] has a maximum absolute error of $5.7 \times 10^{-7}$. Hence the presnet method performs extremely well.

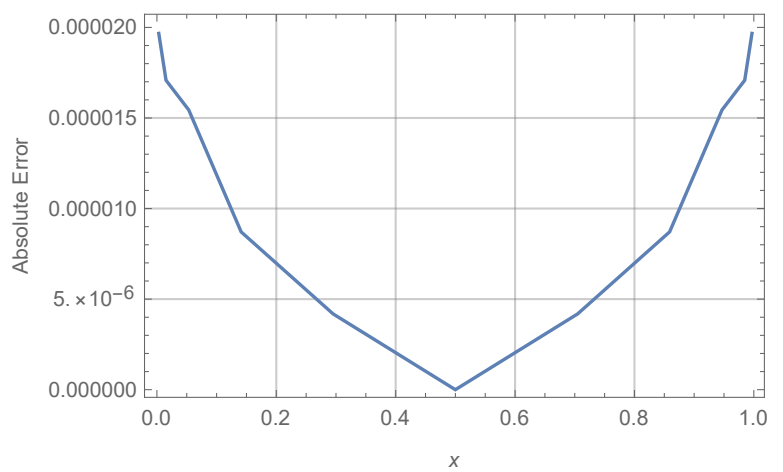

(a)

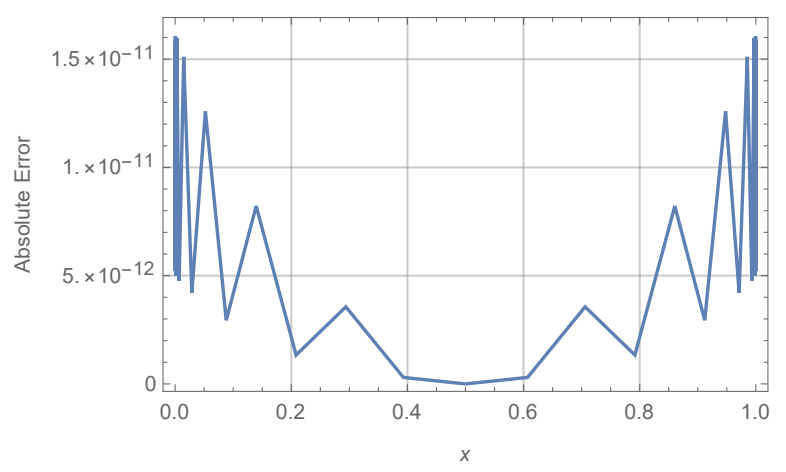

(c)

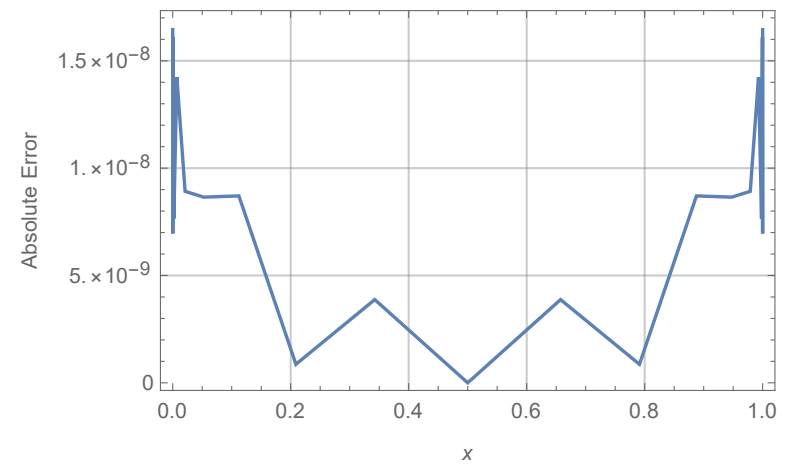

(b)

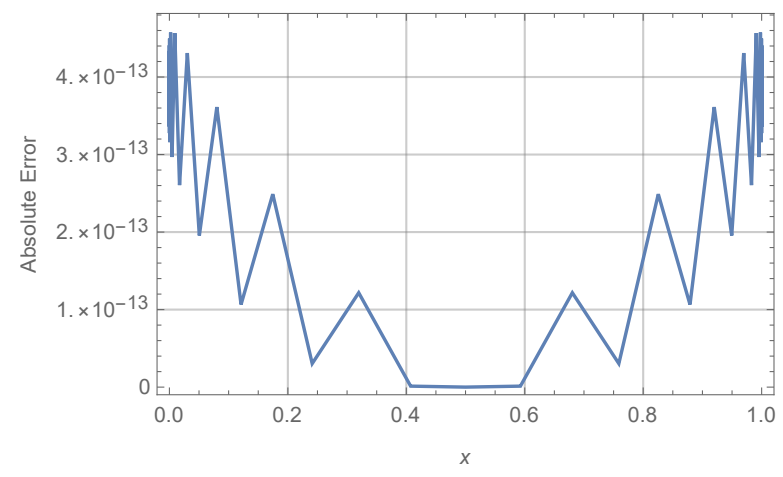

(d)

Figure 4. Plots of the absolute error, for example, 3, using the Sinc-derivative interpolation for various values of $N$. (a) $N=5$; (b) $N=10$; (c) $N=20$; (d) $N=25$. 


\section{Conclusions}

In this paper, the Sinc-derivative collocation methods were used to numerically solve linear and nonlinear multi-point boundary value problems. Sinc based numerical methods are characterized by exponentially decaying errors associated with their approximations which makes them highly efficient.

The Sinc-derivative collocation methods is done by interpolating the first derivative of the unknown variable via Sinc numerical methods and obtaining the desired solution through numerical integration of the interpolation and all higher order derivatives through successive differentiation of the interpolation. Non-homogeneous boundary conditions are reduced to homogeneous case via suitable transformations. The efficiency and the accuracy of the method was demonstrated using illustrative examples which were recently considered using regular Sinc-collocation and other approaches. The result demonstrates the excellent performance of the Sinc-derivative interpolation method.

Author Contributions: Conceptualization, K.A.; Data curation, J.T.; Formal analysis, K.A.; Funding acquisition, K.A.; Investigation, K.A. and J.T.; Methodology, K.A.; Project administration, K.A.; Resources, J.T.; Software, J.T.; Supervision, K.A. All authors have read and agreed to the published version of the manuscript.

Funding: This research received no external funding and the APC was funded by the Qatar National Library.

Conflicts of Interest: The authors declare no conflict of interest.

\section{References}

1. Il'in, V.A.; Moiseev, E.I. Nonlocal boundary value problem of the first kind for a Sturm-Liouville operator in its differential and finite difference aspects. Differ. Equ. 1987, 23, 803-810.

2. Il'in, V.A.; Moiseev, E.I. Nonlocal boundary value problem of the second kind for a Sturm-Liouville operator. Differ. Equ. 1987, 23, 979-987.

3. Gupta, C. Solvability of a three-point nonlinear boundary value problem for a second order ordinary differential equation. J. Math. Anal. Appl. 1992, 168, 540-551. [CrossRef]

4. Ma, R. A survey on nonlocal boundary value problems. Appl. Math. E-Notes 2007, 7, 257-279.

5. Geng, F.; Cui, M. Multi-point boundary value problem for optimal bridge design. Int. J. Comput. Math. 2010, 87, 1051-1056. [CrossRef]

6. Moshinsky, M. Sobre los problems de condiciones a la frontiera en una dimension de carac-teristicas discontinuas. Bol. Soc. Mat. Mexicana 1950, 7, 1-25.

7. Hajji, M.A. Multi-point special boundary-value problems and applications to fluid flow through porous media. In Proceedings of the International Multi-Conference of Engineers and Computer Scientists, Hong Kong, China, 18-20 March 2009.

8. Saadatmandi, A.; Dehghan, M. The use of Sinc-collocation method for solving multi-point boundary value problems. Commun. Nonlinear Sci. Numer. Simul. 2012, 17, 593-601. [CrossRef]

9. Timoshenko, S. Theory of Elastic Stability; Courier Corporation: Chelmsford, MA, USA, 1961.

10. Agarwal, R.P.; Thompson, H.B.; Tisdell, C.C. Three-point boundary value problems for second-order discrete equations. Comput. Math. Appl. 2003, 45, 1429-1435. [CrossRef]

11. Thompson, H.B.; Tisdell, C.C. Three-point boundary value problems for second-order ordinary differential equation. Math. Comput. Model. 2001, 34, 311-318. [CrossRef]

12. Lepin, A.Y.; Ponomarev, V.D. On a positive solution of a three-point boundary value problem. Differ. Equ. 2006, 42, 291-293. [CrossRef]

13. Zhang, Z.G.; Liu, L.S.; Wu, C.X. Nontrival solution of third-order nonlinear eigenvalue problems. Appl. Math. Comput. 2006, 176, 714-721.

14. Pei, M.H.; Chang, S.K. A quasilinearization method for second-order four-point boundary value problems. Appl. Math. Comput. 2008, 202, 54-66. [CrossRef] 
15. Li, X.Y.; Wu, B.Y. A numerical method for solving distributed order diffusion equations. Appl. Math. Lett. 2016, 53, 92-99. [CrossRef]

16. Dehghan, M. Finite difference procedures for solving a problem arising in modeling and design of certain optoelectronic devices. Math. Comput. Simul. 2006, 71, 16-30. [CrossRef]

17. Zou, Y.; Hu, Q.; Zhang, R. On numerical studies of multi-point boundary value problems and its fold bifurcation. Appl. Math. Comput. 2007, 185, 527-537. [CrossRef]

18. Tatari, M.; Dehghan, M. The use of the Adomian decomposition method for solving multi-point boundary value problems. Phys. Scr. 2006, 73, 672-676. [CrossRef]

19. Urabe, M. Numerical solution of multi-point boundary value problems in Chebyshev series theory of the method. Numer. Math. 1967, 9, 341-366. [CrossRef]

20. Lentini, M.; Pereyra, V. A variable order finite difference method for nonlinear multipoint boundary value problems. Math. Comput. 1974, 28, 981-1003. [CrossRef]

21. Ali, J.; Islam, S.; Zaman, G. The solution of multi-point boundary value problems by the optimal homotopy asymptotic method. Comput. Math. Appl. 2010, 59, 2000-2006. [CrossRef]

22. Li, X.; Wu, B. Reproducing kernel method for singular fourth order four-point boundary value problems. Bull. Malays. Math. Sci. Soc. 2011, 34, 147-151.

23. Wu, B.; Li, X. Application of reproducing kernel method to third order three-point boundary value problems. Appl. Math. Comput. 2010, 217, 3425-3428. [CrossRef]

24. Lin, Y.; Cui, M. A numerical solution to nonlinear multi-point boundary value problems in the reproducing kernel space. Math. Methods Appl. Sci. 2011, 34, 44-47. [CrossRef]

25. Li, X.; Wu, B. Reproducing kernel method for singular multi-point boundary value problems. Math. Sci. 2012, 6, 16-20. [CrossRef]

26. Azarnavid, B.; Kourosh, P. An iterative reproducing kernel method in Hilbert space for the multi-point boundary value problems. J. Comput. Appl. Math. 2018, 328, 151-163. [CrossRef]

27. Revelli, R.; Ridolfi, L. Sinc collocation-interpolation method for the simulation of nonlinear waves. Comput. Math. Appl. 2003, 46, 1443-1453. [CrossRef]

28. Abdella, K.; Yu, X.; Kucuk, I. Application of the Sinc method to a dynamic elasto-plastic problem. J. Comput. Appl. Math. 2009, 223, 626-645. [CrossRef]

29. Parand, K.; Dehghan, M.; Pirkhedri, A. Sinc-collocation method for solving the Blasius equation. Phys. Lett. A 2009, 373, 4060-4065. [CrossRef]

30. Parand, K.; Pirkhedri, A. Sinc-Collocation method for solving astrophysics equations. New Astron. 2010, 15, 533-537. [CrossRef]

31. Al-Khaled, K. Numerical approximations for population growth models. Appl. Math. Comput. 2005, 160, 865-873. [CrossRef]

32. Al-Khaled, K. Numerical study of Fisher's reaction-diffusion equation by the Sinc collocation method. J. Comput. Appl. Math. 2001, 137, 245-255. [CrossRef]

33. Winnter, D.F.; Bowers, K.L.; Lund, J. Wind-Driven Currents in a Sea with Variable Eddy Viscosity Calculated via a Sinc-Galerkin Technique. Int. J. Numer. Meth. Fluids 2000, 33, 1041-1073. [CrossRef]

34. Koonprasert, S.; Bowers, K.L. Block matrix Sinc-Galerkin solution of the wind-driven current problem. Appl. Math. Comput. 2004, 155, 607-635. [CrossRef]

35. Stenger, F. Numerical Methods Based on Sinc and Analytic Functions; Springer: Berlin/Heidelberg, Germany, 2012.

36. Lund, J.; Bowers, K.L. Sinc Methods for Quadrature and Differential Equations; Siam: Philadelphia, PA, USA, 1992.

37. Sugihara, M.; Matsuo, T. Recent developments of the Sinc numerical methods. J. Comput. Appl. Math. 2004, 164, 673-689. [CrossRef]

38. Sugihara, M. Near optimality of the sinc approximation. Math. Comput. 2003, 72, 767-786. [CrossRef]

39. Abdella, K. Solving differential equations using Sinc-collocation methods with derivative interpolations. J. Comput. Methods Sci. Eng. 2015, 15, 305-315. [CrossRef]

40. Burden, R.L.; Faires, J.D. Numerical Analysis; Springer: Berlin/Heidelberg, Germany, 2001. 
41. Mohseniahouei, Y.; Abdella, K.; Pollanen, M. The application of the Sinc-Collocation approach based on derivative interpolation in numerical oceanography. J. Comput. Sci. 2015, 7, 13-25. [CrossRef]

42. Abdella, K.; Ross, G.; Mohseniahouei, Y. Solutions to the Blasius and Skiadis problems via sinc-collocation approach. Dyn. Syst. Appl. 2017, 26, 105-120.

43. Sugihara, M. Double exponential transformation in the Sinc-collocation method. J. Comput. Appl. Math. 2002, 149, 7239-7250. [CrossRef]

44. Okayama, T.; Matsuo, T.; Sugihara, M. Improvement of a Sinc-collocation method for Fredholm integral equations of the second kind. BIT Numer. Math. 2011, 51, 339-366. [CrossRef]

45. Rashidinia, J.; Zarebnia, M. Numerical solution of linear integral equations by using sinc-collocation method. Appl. Math. Comput. 2005, 168, 806-822. [CrossRef]

46. Geng, F.Z. A numerical algorithm for nonlinear multi-point boundary value problems. J. Comput. Appl. Math. 2012, 236, 1789-1794. [CrossRef]

47. Geng, F.Z. Solving singular second order three-point boundary value problems using Reproduceing kernel Hilbert space method. Appl. Math. Comput. 2009, 215, 2095-2102.

48. Lie-jun X.; Cai-lian Z.; Song X. A numerical method for third-order non-linear boundary value problems in enginerring. Appl. Math. Comput. 2018, 321, 255-266.

49. Doha, E.H.; Bhrawy, A.H.; Hafez, R.M. On shifted jacobi spectral method for high-order multi-point boundary value problems. Commun. Nonlinear Sci. Numer. Simul. 2012, 17, 3802-3810. [CrossRef]

50. Geng, F.Z.; Qian S.P. A new reproducing kernel method for linear nonlocal boundary value problems. Appl. Math. Comput. 2014, 248, 421-425. [CrossRef]

51. Timizi, I.A.; Twizell, E.H.; Islam, S.U. A numerical method for third-order non-linear boundary value problems in enginerring. Int. J. Comput. Math. 2005, 82, 103-109. [CrossRef]

52. Krajcinvic, D. Sandwitch beam analysis. J. Appl. Mech. 1972, 39, 773-778. [CrossRef]

Publisher's Note: MDPI stays neutral with regard to jurisdictional claims in published maps and institutional affiliations.

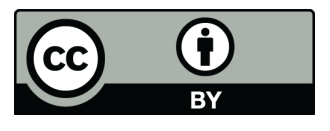

(C) 2020 by the authors. Licensee MDPI, Basel, Switzerland. This article is an open access article distributed under the terms and conditions of the Creative Commons Attribution (CC BY) license (http:/ / creativecommons.org/licenses/by/4.0/). 